\section{Incentivo ao aleitamento materno: uma avaliação das equipes de saúde da família do município de Olinda, Pernambuco}

\section{Incentive to breast feeding: an evaluation of family health teams in the municipality of Olinda, Pernambuco}

Luciana Santos Dubeux 1

Paulo Germano de Frias 2

Suely Arruda Vidal 3

Daniela Maria dos Santos 4

1-4 Instituto Materno Infantil de Pernambuco, IMIP. Rua dos Coelhos, 300. Recife, PE, Brasil. CEP: 50.070-550. Tel / Fax: +55 +81 2122.4141

\begin{abstract}
Objectives: to assess performance of the Breast Feeding Incentive Program implementation in the municipality of Olinda, in 2003.

Methods: a normative, cross sectional assessment study in the 40 Family Healthcare Teams implemented in two Sanitary Districts of the Olinda municipality from July to September, 2003. This study focused on the Breast Feeding Incentive Program and determined as implemented (90\% to $100 \%$ of affirmative answers), partially acceptable implementation (70\% to less than 90\% of affirmative answers) and not implemented (less than 50\% of affirmative answers).

Results: the Breast Feeding Program implementation program by the Family Healthcare Teams was rated in $22.5 \%$, considering partially acceptable implementation with the best performance determined for Sanitary District II. Among activities to promote the program, (highlights) the following was listed "early incentive to exclusive breast feeding", "prenatal orientation", "and nutritional orientation for weaning" and "multidisciplinary professional involvement", as being especially focused by the teams of both Sanitary Districts.

Conclusions: insufficient adhesion of family healthcare teams to the activities established by the Ministry of Health, impacting the degree of implementation of the Breast Feeding Incentive Program and possibly not favoring prevalence increase of exclusive breast feeding and modalities in the municipality of Olinda.
\end{abstract}

Key words Breast feeding, Quality assurance, health care, Program evaluation, Family health

\section{Resumo}

Objetivos: avaliar o grau de implantação da ação de Incentivo ao Aleitamento Materno (IAM) em Olinda, 2003.

Métodos: estudo avaliativo-normativo, transversal realizado nas 40 Equipes de Saúde da Família (ESF) implantadas nos dois Distritos Sanitários (DS) de Olinda, entre julho e setembro de 2003. O instrumento utilizado contemplou questões relativas à ação de IAM e a julgou como implantada $(90 \%$ a $100 \%$ de respostas positivas), parcialmente implantada aceitável (70\% a menos de $90 \%$ de respostas positivas), parcialmente implantada não aceitável (50\% a menos de $70 \%$ de respostas positivas) e não implantada (concordância inferior a 50\%).

Resultados: a ação de IAM foi implantada em 22,5\% das ESF e em 57,5\% parcialmente implantada aceitável, havendo melhor desempenho do DS II. Dentre as atividades para a promoção da ação, destacam-se: o "incentivo precoce ao aleitamento materno exclusivo", a "orientação no pré-natal", a "orientação alimentar para o desmame" e o "envolvimento multiprofissional", como as mais desenvolvidas pelas equipes de ambos os DS.

Conclusões: houve insuficiente adesão das ESF às atividades preconizadas pelo Ministério da Saúde, repercutindo no grau de implantação da ação de IAM, e possivelmente desfavorecendo um aumento da prevalência do aleitamento materno exclusivo e suas modalidades em Olinda.

Palavras-chave Aleitamento materno, Garantia da qualidade dos cuidados de saúde, Avaliação de programas, Saúde da família 


\section{Introdução}

Torna-se relevante a constante abordagem ao tema aleitamento materno, devido a sua complexidade e importância para o desenvolvimento da Saúde Infantil. A prática da amamentação, principalmente a exclusiva até seis meses de vida, traz benefícios que envolvem: o efeito protetor contra alergias alimentares, as doenças diarréicas e infecções comuns à infância, ${ }^{1} \mathrm{o}$ adequado desenvolvimento crânio-facial e conseqüente prevenção de alterações de fonação, deglutição e respiração, 2 além de desempenhar papel importante no desenvolvimento intelectual e no relacionamento afetivo das mães com os seus bebês. ${ }^{3}$

Entretanto, apesar da importância dessa prática, é de amplo conhecimento a existência de fatores que se interpõem ao efetivo desenvolvimento da mesma, tais como a atuação dos serviços de saúde, a educação materna, a classe socioeconômica, as crenças relacionadas com o aleitamento, o apelo da indústria para o uso de leite e bicos artificiais 3,4 e o retorno precoce das nutrizes ao trabalho.

Como resultado desses fatores foram identificadas baixas taxas de prevalência do aleitamento materno (AM) em todas as capitais das regiões do Brasil em 1999.5 No Nordeste, a prevalência de aleitamento materno exclusivo (AME) em menores de quatro meses foi de $37,8 \%$ e para a cidade do Recife, 27,4\%.6 Em Olinda, de acordo com o Sistema de Informações da Atenção Básica (SIAB), a prevalência do AME, para as áreas cobertas pelo Programa de Agente Comunitário de Saúde e de Saúde da Família, foi de $57,2 \%$ em 2000.7

Para fazer frente ao desafio de reverter esse quadro são necessários a implementação de atividades educativas e o envolvimento institucional. Nesse contexto, a Estratégia Saúde da Família, é um cenário pertinente para realização desse trabalho, haja vista que a Norma Operacional de Assistência à Saúde do Sistema Único de Saúde (NOAS - SUS 01/2001) destaca a promoção ao Aleitamento Materno como uma das ações mínimas para a Atenção Básica à Saúde da Criança. ${ }^{8}$

Reforçando a estratégia de promoção ao aleitamento materno na atenção básica, foram definidos, em 2001, os 10 passos para a Unidade Básica Amiga da Amamentação 9 e em 2003 foi apresentada a adaptação desses passos para as Unidades de Saúde da Família. ${ }^{10}$ Eles concordam e complementam a Iniciativa Hospital Amigo da Criança, criada em 1992, para desenvolvimento da atenção humanizada11 e contém 10 passos para o apoio, proteção e promoção ao aleitamento nas maternidades. 4,12

Considerando que o Ministério da Saúde estimu- la a promoção, proteção e apoio ao AM e que a Estratégia Saúde da Família está em processo crescente de expansão e qualificação, torna-se imprescindível o acompanhamento sistemático das atividades relacionadas a essa ação. Nesse contexto, o presente estudo se propõe a realizar uma avaliação do grau de implantação da ação de Incentivo ao Aleitamento Materno nos dois distritos sanitários do município de Olinda.

\section{Métodos}

Neste estudo, foram avaliadas as 40 Equipes de Saúde da Família (ESF) implantadas e em funcionamento no período de julho a setembro de 2003, e pertencentes aos dois Distritos Sanitários (I e II) do município de Olinda.

Localizando-se na região metropolitana de Recife, litoral norte do estado de Pernambuco, Olinda está habilitada na gestão plena do sistema e a rede básica de saúde e dispunha, no período de estudo, de 56 unidades prestadoras de serviços ambulatoriais, dos tipos público e filantrópico. No processo de reordenamento da rede alguns Centros de Saúde têm sido transformados em Unidades de Saúde da Família (USF).

Realizou-se um estudo descritivo de corte transversal, do tipo avaliativo-normativo, que abordou a apreciação do processo, especificamente a dimensão técnica dos serviços e da estrutura para avaliar o grau de implantação da ação de Incentivo ao Aleitamento Materno.

Utilizou-se para a coleta de dados um instrumento adaptado por técnicos do Instituto Materno Infantil de Pernambuco (IMIP) às normas e diretrizes da estratégia Atenção Integral às Doenças Prevalentes na Infância (AIDPI), 13 que foi previamente validado e utilizado em estudos anteriores.14,15 Esse instrumento contém questões relativas às atividades preconizadas para a promoção ao aleitamento materno e orientação para o desmame e também referente à existência de normas e de material educativo.

Como critério de julgamento preconizou-se que a proporção das respostas afirmativas às perguntas formuladas corresponderia ao grau de implantação obtido por cada ESF, não existindo critérios de peso entre as questões. Dessa forma, considerou-se inicialmente a ação como: implantada (quando todas as questões fossem respondidas afirmativamente), parcialmente implantada, (se houvesse concordância com $50 \%$ a $90 \%$ das respostas preconizadas) e não implantada, (se tivesse menos de $50 \%$ de concordância). 
Entretanto, com a adoção desses pontos de corte bastava uma questão ser discordante para atribuir-se o grau de parcialmente implantada, ficando com uma margem muito ampla e o instrumento muito rígido no seu julgamento. Nesse contexto, os pontos de corte foram adaptados para melhor expressar a realidade, passando a ser considerada: implantada $(90 \%$ a $100 \%$ de respostas positivas), parcialmente implantada aceitável (70\% a menos de $90 \%$ de respostas positivas), parcialmente implantada não aceitável (50\% a menos de $70 \%$ de respostas positivas) e não implantada (concordância inferior a 50\%).

$\mathrm{O}$ instrumento foi respondido por médicos ou enfermeiros das Equipes de Saúde da Família, selecionados de acordo com a disponibilidade dos mesmos no momento da entrevista.

Para avaliar a confiabilidade das informações fornecidas, procedeu-se o sorteio de $10 \%$ do universo das equipes e repetiu-se a entrevista, tendo sido constatado um percentual de concordância de $75 \%$ para o grau de implantação da ação nos dois períodos de entrevista.

Quanto à concordância intra-atividades houve uma variação de 25 a $100 \%$, sendo as atividades "normas do Ministério da Saúde", "material educativo para o aleitamento materno", "desenvolvimento de práticas educativas" e "entrosamento com entidades de apoio ao aleitamento materno" as responsáveis pelo menor percentual de concordância.

Os dados foram processados no programa Microsoft Excel/Windows, sendo as informações expressas sob a forma de proporção e apresentadas em tabelas de distribuição simples de freqüência.

\section{Resultados}

A avaliação indicou que embora apenas $22,5 \%$ das ESF do município de Olinda tenham apresentado a ação de IAM implantada, uma parcela considerável se classificou como parcialmente implantada aceitável $(57,5 \%)$ e nenhuma equipe como não implantada. (Tabela 1)

Ainda na Tabela 1, analisando-se os Distritos Sanitários separadamente, observou-se discreta vantagem do DS II em relação ao DS I, já que os mesmos tiveram respectivamente $26,7 \%$ e $20,0 \%$ de suas ESF com a ação implantada. Além disso, o DS II teve menos equipes na condição parcialmente implantada não aceitável $(13,4 \%)$ quando comparado ao DS I (24,0\%). Ressaltam-se ainda melhores percentuais do DS II para as equipes classificadas como parcialmente implantada aceitável $(60,0 \%)$, enquanto o DS I teve $56,0 \%$ das ESF nessa condição.

No tocante ao cumprimento das atividades necessárias para implantação da ação em questão destacam-se: o incentivo precoce ao aleitamento materno exclusivo, a orientação no pré-natal, a orientação alimentar para o desmame e o envolvimento multiprofissional, que foram respondidas afirmativamente por todas as equipes de ambos os distritos sanitários de Olinda. Entretanto, atividades igualmente importantes para a promoção da ação tais como "material educativo para o desmame" e "entrosamento com entidades de apoio ao aleitamento materno" foram identificadas em menor percentual no DS I $(20,0 \%$ e $12,0 \%)$ e no DS II $(33,4 \%$ e $33,4 \%$ ), observando-se melhores resultados para o DS II em relação ao DS I. (Tabela 2)

Tabela 1

Distribuição da freqüência do grau de implantação da ação de Incentivo ao Aleitamento Materno. Olinda, Pernambuco, 2003.

\begin{tabular}{|c|c|c|c|c|c|c|}
\hline \multirow{2}{*}{ Grau de implantação } & \multicolumn{2}{|c|}{ DS I } & \multicolumn{2}{|c|}{ DS II } & \multicolumn{2}{|c|}{ Olinda } \\
\hline & $\mathbf{n}$ & $\%$ & $\mathbf{n}$ & $\%$ & $\mathbf{n}$ & $\%$ \\
\hline Implantada & 5 & 20,0 & 4 & 26,7 & 9 & 22,5 \\
\hline Parcialmente implantada aceitável & 14 & 56,0 & 9 & 60,0 & 23 & 57,5 \\
\hline Parcialmente implantada não aceitável & 6 & 24,0 & 2 & 13,4 & 8 & 20,0 \\
\hline Não implantada & - & - & - & - & - & - \\
\hline Total & 25 & 100,0 & 15 & 100,0 & 40 & 100,0 \\
\hline
\end{tabular}

DS = Distrito sanitário 
Tabela 2

Distribuição da freqüência das atividades referentes à ação de Incentivo ao Aleitamento Materno nas equipes de saúde da família de acordo com os distritos sanitários. Olinda, Pernambuco, 2003.

\begin{tabular}{|c|c|c|c|c|c|c|}
\hline \multirow{2}{*}{ Grau de implantação } & \multicolumn{2}{|c|}{ DS I $(N=25)$} & \multicolumn{2}{|c|}{ DS II $(N=15)$} & \multicolumn{2}{|c|}{ Olinda $(\mathrm{N}=40)$} \\
\hline & n & $\%$ & n & $\%$ & $\mathbf{n}$ & $\%$ \\
\hline Incentivo precoce ao aleitamento materno exclusivo & 25 & 100,0 & 15 & 100,0 & 40 & 100,0 \\
\hline Orientação no pré-natal & 25 & 100,0 & 15 & 100,0 & 40 & 100,0 \\
\hline Orientação alimentar para o desmame & 25 & 100,0 & 15 & 100,0 & 40 & 100,0 \\
\hline Normas do Ministério da Saúde & 17 & 68,0 & 08 & 53,3 & 25 & 62,5 \\
\hline Atividade diferenciada para dificuldades no aleitamento materno & 24 & 96,0 & 15 & 100,0 & 39 & 97,5 \\
\hline Material educativo para o aleitamento materno & 17 & 68,0 & 13 & 86,7 & 30 & 75,0 \\
\hline Material educativo para o desmame & 05 & 20,0 & 05 & 33,4 & 10 & 25,0 \\
\hline Desenvolvimento de práticas educativas & 19 & 76,0 & 13 & 86,7 & 32 & 80,0 \\
\hline Entrosamento com entidades de apoio ao aleitamento materno & 03 & 12,0 & 05 & 33,4 & 08 & 20,0 \\
\hline Envolvimento multiprofissional & 25 & 100,0 & 15 & 100,0 & 40 & 100,0 \\
\hline
\end{tabular}

Outras atividades como "normas do Ministério da Saúde" e "atividade diferenciada para dificuldades no aleitamento materno" apresentaram variações de $68,0 \%$ (DS I) a $53,3 \%$ (DS II) para a primeira e $96,0 \%$ (DS I) a $100,0 \%$ (DS II) para a segunda. As atividades "desenvolvimento de práticas educativas" e "material educativo para o desmame" apresentaram maior proporção no DS II. A adesão foi de $76,0 \%$ no DS I e $86,7 \%$ no DS II para o "desenvolvimento de práticas educativas". (Tabela 2)

\section{Discussão}

A avaliação normativa representa uma ferramenta para o monitoramento, já que consiste em se fazer um julgamento sobre uma intervenção comparando os recursos empregados e sua organização (avaliação de estrutura), os serviços ou os bens produzidos (avaliação de processo) e os resultados obtidos, de acordo com critérios e normas pré-estabelecidas, 16 sendo útil para melhoria da qualidade dos serviços de saúde. ${ }^{17}$ Destaca-se ainda, a importância da avaliação normativa para análise da implantação de ações 18 como a de IAM.

O primeiro estudo no estado de Pernambuco aplicando-se a metodologia da presente avaliação foi realizado em 1995. Foram observadas 44,6\% e $66,7 \%$ de unidades de saúde com a ação "Incentivo ao Aleitamento Materno" implantada em Pernambuco e Recife, respectivamente. ${ }^{18}$ Entretanto, em 2002, utilizando a mesma metodologia, nenhuma das unidades de atenção básica de Recife em funcionamento apresentou a ação implantada. 14

Já Oliveira et al.,17 ao realizarem uma avaliação no estado do Rio de Janeiro, elegeram como "boa" a unidade de atenção básica que apresentasse suporte rotineiro ao aleitamento materno para gestantes e puérperas e tivesse grande parte da equipe treinada para essa ação. Entretanto, não considerou nenhuma unidade nessa classificação.

Observa-se, então, a importância de uma intensa compreensão do conjunto de atividades desenvolvidas nas Unidades de Saúde para análise e articulação dos vários momentos do processo de trabalho, ${ }^{19}$ possibilitando uma coerente avaliação da ação IAM. Assim, foram avaliadas atividades preconizadas para a implantação da ação em estudo, conforme discutidas a seguir.

$\mathrm{O}$ incentivo precoce ao aleitamento materno exclusivo, representado pela transmissão às mães e familiares sobre a importância do início da prática nas primeiras horas de vida, ${ }^{4}$ foi afirmado pelo universo dos entrevistados, ressaltando o valor atribuído pelos profissionais a um dos passos primários para o sucesso da ação. Da mesma forma, a orientação no pré-natal que apresenta importante efeito na amamentação, principalmente se houver a orientação sobre técnicas e o fortalecimento da autoconfiança da gestante, ${ }^{4}$ foi descrita com unanimidade.

Em relação à orientação alimentar para o desmame, Kitoco et al. 20 observaram que apenas $32,2 \%$ 
das crianças amamentadas em Florianópolis, Santa Catarina, e 24,8\% em João Pessoa, Paraíba, receberam alimentos semi-sólidos/sólidos na faixa etária de seis a nove meses, conforme recomendação da Organização Mundial de Saúde. Nesse estudo, embora não tenha sido questionado e/ou checado o procedimento para orientação ao desmame, a totalidade dos entrevistados responderam afirmativamente a essa questão.

A utilização de normas do Ministério da Saúde para promoção, proteção e apoio ao aleitamento, procedimento essencial para permanência de uma memória institucional e para a prática efetiva da amamentação, vem sendo ressaltada em Iniciativas como o Hospital Amigo da Criança, 11,12,21 Iniciativa Unidade Básica Amiga da Amamentação 9,10 tendo sido ainda sumarizada como passo fundamental por Oliveira et al. ${ }^{17} \mathrm{Na}$ presente avaliação, 62,5\% dos entrevistados indicaram haver normas escritas em suas unidades; entretanto, destacando-se o fato de não fazer parte desse método a checagem do material, e observando-se o grau de implantação da ação de IAM em todo município, cabe questionar se as normas, caso presentes, são cumpridas ou se estão adequadas à realidade institucional local.

A realização de atividades diferenciadas para dificuldades no aleitamento materno acontece rotineiramente em $97,5 \%$ das equipes e a maioria dessas as faz por meio de visitas domiciliares. Essa ocorrência, conhecida como "dificuldade de amamentar", inclui-se nas principais causas de desmame precoce. Assim, cabe não apenas aos amigos e familiares, mas também fundamentalmente as unidades básicas de saúde o apoio necessário nessa ocasião. 4

Destaca-se que o conhecimento sobre as vantagens do aleitamento é um dentre os vários fatores que influenciam as intenções de amamentar, mas isoladamente pode não ser suficiente, pois conforme Graffy,22 a decisão das mulheres de como alimentar seus bebês ocorre antes da gravidez. Assim, ressaltando que as gestantes podem ser fortemente influenciadas por familiares e amigos 23 torna-se vital o fortalecimento de um apoio social em prol do aleitamento materno. Para isso, sugere-se a participação de parceiros, amigos e familiares em sessões educativas, que terão valia para fortalecer a confiança e habilidade das mães em relação à prática. ${ }^{4}$

No presente estudo, $76 \%$ dos entrevistados dos DS I e $86,7 \%$ do DS II mencionaram o desenvolvimento de práticas educativas para promoção ao aleitamento e para outras questões referentes aos cuidados com a gestante e a criança, durante a gravidez. Portanto, como a maioria dos profissionais afirmou não ter uma rotina para promoção da ação
IAM de forma exclusiva, a atividade "desenvolvimento de práticas educativas" foi considerada implantada mesmo se o assunto estivesse incluído nas práticas educativas quinzenais durante o pré-natal. Foi questionada ainda às equipes a posse de material educativo para o aleitamento materno e para o desmame, obtendo-se o percentual de $75 \%$ e $25 \%$ respectivamente. Observou-se na entrevista a frustração de alguns profissionais pela ausência do material, embora seja tendência que a disseminação do paradigma da educação popular em saúde, baseado na construção do conhecimento a partir de saberes profissionais e populares na perspectiva da integralidade das ações em saúde, 24 dispensaria o material em prol da confecção dos mesmos pelo grupo educativo.

No tocante ao entrosamento com entidades de apoio para o aleitamento materno, observou-se que apenas $20 \%$ das equipes têm relação com tais entidades, sendo essa atividade talvez uma das principais responsáveis pela não implantação da ação de IAM. Barros 25 constatou forte relação entre o comparecimento aos centros de lactação e o aleitamento exclusivo até os quatro meses.

Ainda como atitude necessária e fundamental para elevar a prevalência da amamentação destacase o envolvimento multiprofissional na sua promoção,26 havendo, nesse estudo, o universo das equipes avaliadas trabalhando dessa forma. Observa-se que a atuação na estratégia saúde da família, cujos princípios envolvem a realização de atividades multiprofissionais em prol da integralidade da atenção a saúde, provavelmente favoreceu esse quadro. Entretanto, deve-se ressaltar que o cirurgiãodentista, profissional integrante da equipe de saúde da família desde o ano 200027 e necessário para orientação quanto às vantagens do aleitamento sobre o desenvolvimento das estruturas e funções do sistema estoma-tognático, 28 embora excluído da entrevista, não foi listado como profissional envolvido na ação.

Afirma-se, por fim, que para a real implantação da ação de IAM é necessária uma maior adesão às atividades antes descritas, tendo, cada uma dessas, valor singular para a promoção da ação, sendo a realização do conjunto das atividades, fundamental para reverter as baixas taxas de prevalência do aleitamento materno, principalmente o exclusivo. Assim, conclui-se que mediante os resultados observados, com proporção de implantação da ação inferior a 50\% das equipes, há grande necessidade de discussão e sensibilização dos profissionais sobre a importância da ação de Incentivo ao Aleitamento Materno para consequiente melhoria da sua aplicação no cotidiano dos serviços de saúde. 


\section{Agradecimentos}

Os autores agradecem aos profissionais das equipes de Saúde da Família e da Secretaria Municipal de Saúde da Prefeitura de Olinda.

\section{Referências}

1. Passos MC, Lamounier JA, Silva CAM. Práticas de amamentação no município de Ouro Preto, MG, Brasil. Rev Saúde Pública 2000; 34: 617-22.

2. Neiva FCB, Cattoni DM, Ramos JLA, Issler H. Desmame precoce: implicações para o desenvolvimento motororal. J Pediatr [Rio de Janeiro] 2003; 79: 7-12.

3. Toma TS, Monteiro CA. Avaliação da promoção do aleitamento materno nas maternidades públicas e privadas do município de São Paulo. Rev Saúde Pública 2001; 35: 409-14.

4. OMS (Organização Mundial de Saúde). Evidências científicas dos dez passos para o sucesso no aleitamento materno. Brasília (DF): A Organização; 2001.

5. UNICEF (Fundo das Nações Unidas para a Infância). Saúde e nutrição das crianças nordestinas: pesquisa estaduais 1987-1992. Brasília (DF): O Fundo; 1995.

6. Ministério da Saúde. Pesquisa de prevalência do aleitamento materno nas capitais e no Distrito Federal. Brasília (DF) O Ministério; 2001

7. Ministério da Saúde. FUNASA (Fundação Nacional de Saúde). SIAB (Sistema de Informações de Atenção Básica). Disponível em: http://www.aleitamento.org.br/indicedeam/ pernambuco.html [2003 nov 11]

8. Ministério da Saúde. DEAB (Departamento de Atenção Básica). A regionalização e a atenção básica - desafios da NOAS-SUS 01/2001 e os instrumentos que oferece para reorganizar o sistema. Saúde Fam 2002; (4): 11-16.

9. Carvalho MR. Dez passos para o sucesso do aleitamento materno da Iniciativa Unidade Básica Amiga da Amamentação, 2003. Disponível em: http://www.aleitamento.med.br [2003 Set 20]

10. Aquino RGP, Villasbôas AL, Carvalho EMR, Lima HCA. Uma dúzia de passos para a Unidade de Saúde da Família Amiga da Amamentação. In: VIII Encontro Nacional de Aleitamento Materno; 2003 nov.; Cuiabá. Disponível em: http://www.aleitamento.med.br. [2004 out 9].

11. Ministério da Saúde. Secretaria de Políticas de Saúde. O desafio de construir e implementar políticas de saúde: relatório de gestão 2000-2002. Brasília (DF): O Ministério 2002

12. Lamounier JA. Promoção e incentivo ao aleitamento materno: Iniciativa Hospital Amigo da Criança. J Pediatr [Rio de Janeiro] 1996; 72: 363-8.

13. Atenção integrada às doenças prevalentes na infância.In: Curso de capacitação, módulo de Introdução; 1995; Brasília. Brasília (DF): Ministério da Saúde, OMS e OPS; 1999.

14. Bezerra LCA. Incentivo ao aleitamento materno e orientação alimentar para o desmame: o grau de implantação da ação nos centros de saúde e equipes de saúde da família da cidade do Recife, 2002 [residência]. Recife: Departamento de Saúde Coletiva, Centro de Pesquisas Aggeu Magalhães, Fundação Oswaldo Cruz; 2002.

15. Macedo VC. Avaliação normativa do programa de assistência integral à saúde da criança nas equipes de saúde da família do município do Recife, 2002 [residência]. Recife: Departamento de Saúde Coletiva, Centro de Pesquisas Aggeu Magalhães, Fundação Oswaldo Cruz; 2002.

16. Contrandriopoulos A-P, Champagne F, Denis J, Pineault R. A avaliação na área de saúde: conceitos e métodos. In Hartz ZMA, organizadora. Avaliação em saúde: dos modelos conceituais à prática na análise da implantação de programas. Rio de Janeiro: FIOCRUZ; 1997. p. 29-47.

17. Oliveira MIC, Camacho LAB, Tedstone AE. A method for the evaluation of primary health care units'practice in the promotion, protection, and support of breastfeeding: results from de state of Rio de Janeiro, Brasil. J Hum Lact 2003; 19: 365-73.

18. Vidal SA, Frias PG, Marques NM. Avaliação normativa do Programa de Atenção à Criança (PAISC) em Pernambuco. Rev Bras Saúde Matern Infant 2001; 1: 129-35.

19. Sala A, Nemes MIB, Cohen, D.D. A avaliação na prática programática. In: Schraiber LB, Nemes MIB, Gonçalves RBM, organizadores. Programas e ações na unidade básica. 2. ed. São Paulo: HUCITEC; 2000. p. 22-9.

20. Kitoco PM, Rea MF, Venancio SI, Vasconcelos ACCP, Santos EKA, Monteiro CA. Situação do aleitamento materno em duas capitais brasileiras: uma análise comparada. Cad Saúde Pública 2000; 16: 1111-9.

21. WHO (World Health Organization). Indicators for assessing breastfeeding practices. Genebra: The Organization; 1997.

22. Graffy JP. Mother's attitudes to and experience of breastfeeding: a primary care study. Br J Gen Pract 1992; 42: 61-4.

23. Giugiani ERJ. Effect of breastfeeding support from different souces on mother's decisions to breastfeed. J Hum Lact 1994; 10: 157-61.

24. Sophia D. Os homens se educam entre si, mediatizados pelo mundo. Tema 2001; (21): 2-6.

25. Barros FC. The impact of lactation centres on breastfeeding patterns, morbidity and growth, a birth short study. Acta Pediatr 1995; 11: 88-92.

26. Rea MF. O pediatra e a amamentação exclusiva. J Pediatr [Rio de Janeiro] 2003; 79: 479-80.

27. Ministério da Saúde. Portaria n ${ }^{\circ} 1444 / G M$ de 28 de dezembro de 2000. Disponível em: http://portalweb02.saude. gov.br/saude/area.cfm?id_area=169. [2003 dez 18].

28. Carvalho GD. Amamentação: prevenção primária das alterações morfofuncionais comuns da Síndrome do Respirador Bucal. São Paulo: Lovise; 2003. p. 205-40.

Recebido em 15 de julho de 2004

Versão final apresentada em 29 de setembro de 2004

Aprovado em 30 de outubro de 2004 